\title{
Evidence for autism spectrum disorder in Jacobsen syndrome: identification of a candidate gene in distal 11q
}

\author{
Natacha Akshoomoff, PhD', Sarah N. Mattson, $\mathrm{PhD}^{2}$ and Paul D. Grossfeld, MD³
}

Purpose: Jacobsen syndrome, also called the 11q terminal deletion disorder, is a contiguous gene disorder caused by the deletion of the end of the long arm of chromosome 11. Intellectual skills range from low average to severe/profound intellectual disability and usually correlate with deletion size. Comprehensive genotype/phenotype evaluations are limited, and little is known about specific behavioral characteristics associated with $11 \mathrm{q}$ terminal deletion disorder.

Methods: In this prospective study, 17 patients with $11 \mathrm{q}$ terminal deletion disorder underwent cognitive and behavioral assessments. Deletion sizes were determined by array comparative genomic hybridization.

Results: Deletion sizes ranged from 8.7 to $14.5 \mathrm{Mb}$ across the patients. We found that 8 of 17 patients (47\%) exhibited behavioral characteristics consistent with an autism spectrum disorder diagnosis. There was no correlation between deletion size and the presence of autism spectrum disorder, implicating at least one predisposing gene in the distal $8.7 \mathrm{Mb}$ of 11q. The findings from three additional patients with autistic features and "atypical" distal 11q deletions led to the identification of an autism "critical region" in distal 11q containing four annotated genes including ARHGAP32 (also known as RICS), a gene encoding rho GTPase activating protein.

Conclusion: Results from this study support early autism spectrum disorder screening for patients with $11 \mathrm{q}$ terminal deletion disorder and provide further molecular insights into the pathogenesis of autism spectrum disorder.

Genet Med advance online publication 24 July 2014

Key Words: autism spectrum disorder; ARHGAP32; behavioral phenotype; Jacobsen syndrome; 11q terminal deletion

\section{INTRODUCTION}

Jacobsen syndrome (JBS; MIM 147791), also called the 11q terminal deletion disorder (11q-), is a contiguous gene disorder caused by the deletion of the end of the long arm of chromosome $11 .^{1-3}$ There is considerable phenotypic variability, but common problems include dysmorphic features, intellectual disability, thrombocytopenia, congenital heart defects, pyloric stenosis, and structural renal anomalies..$^{2-6}$ Our previous study demonstrated a range of intellectual impairment across 14 patients with $11 \mathrm{q}$ - and a strong correlation between deletion size and degree of cognitive impairment. ${ }^{7}$ These results also implicated at least two loci in distal $11 \mathrm{q}$ contributing to the cognitive deficits in 11q-.

There have been case reports of autistic-like features in 11qpatients, ${ }^{8-10}$ but there are no systematic prospective studies using standard research diagnostic procedures. The present study is a prospective evaluation of 11q- patients with two aims: (i) to determine the likelihood that patients meet criteria for autism spectrum disorder (ASD) using standardized diagnostic procedures and (ii) to identify the candidate gene(s) responsible for this phenotype.

\section{MATERIALS AND METHODS}

\section{Subjects}

There were 5 male and 12 female patients with ages ranging from 3 to 21 years at the time of testing. All participants $(n=$ 17) were prospectively recruited from the $11 \mathrm{q}$ Research and Resource Group, which has hosted a biennial conference in San Diego, California, since 1998. Most testing was conducted in conjunction with this conference. Before participation, written informed consent (parents) and assent (children older than age 7 years) was obtained, as approved by the institutional review board.

Patients with a diagnosis of JBS by previous karyotype analysis or array comparative genomic hybridization $(\mathrm{aCGH})$ were eligible for participation in the study. We defined JBS as a constellation of clinical features in association with a terminal 11q deletion that includes the ETS-1 and FLI-1 genes. These two genes likely cause the two historically life-threatening aspects of the syndrome: congenital heart disease and Paris-Trousseau bleeding disorder. ${ }^{2,3,11,12}$ Only patients with pure $11 \mathrm{q}$ terminal deletions were included in the main cohort. We excluded patients with unbalanced translocations (deletion of distal 11q

\footnotetext{
All authors contributed equally to this work.

${ }^{1}$ Department of Psychiatry, School of Medicine, University of California, San Diego, San Diego, California, USA; ${ }^{2}$ Department of Psychology, San Diego State University, San Diego, California, USA; ${ }^{3}$ Department of Pediatrics, School of Medicine, University of California, San Diego, San Diego, California, USA. Correspondence: Natacha Akshoomoff (nakshoomoff@ucsd.edu)
} 
and duplication of another locus) inherited from a parent carrying a balanced translocation. As expected, the majority of patients have congenital heart defects, some of which have been surgically repaired.

Three additional patients with atypical deletions (i.e., interstitial deletions in distal 11q or smaller terminal deletions not spanning the ETS-1 and FLI-1 genes), two of whom had a previously known diagnosis of ASD, are also described but not included in the main cohort. Specifically, patient 18 initially had autism diagnosed and subsequently underwent aCGH analysis that identified a maternally inherited $6.1-\mathrm{Mb}$ terminal deletion in 11q. Patient 19 had many clinical features of JBS, including intellectual disability, congenital heart disease, and Paris-Trousseau bleeding disorder. Subsequent aCGH analysis identified a de novo 7.3-Mb interstitial deletion that spanned the ETS- 1 and FLI- 1 genes. Patient 20 was originally identified in the UNIQUE database (http://www.rarechromo.org; patient ID 16270). This patient originally underwent aCGH testing because of intellectual disability and mild dysmorphic features, which identified a de novo approximately $0.3-\mathrm{Mb}$ interstitial deletion in distal $11 \mathrm{q}$ that was deleted in all 17 patients in our cohort and in the two other patients with atypical deletions described.

\section{Measures}

Parent questionnaires and interviews. Before testing, parents completed a demographic and developmental history questionnaire. Parents also completed the Social Communication Questionnaire, ${ }^{13}$ which is a 40 -item parent report screening measure that provides a dimensional measure of ASD symptomatology. The Social Communication Questionnaire has good sensitivity and specificity with respect to the separation of ASD from non-ASD diagnoses at all intelligence quotient levels. Children under 5 years of age who obtain a score of 11 or more points and children 5 years of age and older who obtain a score of 15 or more points are considered to be highly likely to meet criteria for an ASD. ${ }^{14}$

On the day of testing, parents completed the Vineland Adaptive Behavior Scales, Second Edition Survey Interview form (VABS-II) with a trained examiner. ${ }^{15}$ The VABS-II is a parent interview measure of child adaptive behavior skills and provides a standardized assessment of adaptive behavior in three subdomains (communication, socialization, and daily living skills) and an overall adaptive behavior composite score.

Behavioral testing. General cognitive levels were estimated using a step-down procedure. ${ }^{16}$ One of four measures was used to estimate general ability levels based on the child's chronological age and developmental level at the time of testing: the Bayley Scales of Infant and Toddler Development, Third Edition (Bayley-III) ${ }^{17}$; the Differential Ability Scales; ${ }^{18}$ the Wechsler Intelligence Scale for Children, Fourth Edition; ${ }^{19}$ or the Wechsler Adult Intelligence Scale, Fourth Edition..$^{20}$

All subjects in the prospective cohort and one with an atypical deletion were administered the Autism Diagnostic Observation
Schedule (ADOS). ${ }^{21}$ The ADOS is a standardized observation of social behavior in naturalistic and communicative contexts, with different modules and tasks for children of different ages and language levels. The ADOS was specifically designed to assist with the diagnosis of ASD. The revised algorithm of the ADOS (ADOS-2) $)^{22,23}$ was used for classification purposes. This revised algorithm includes social, communication, and restricted repetitive behaviors and thus better represents all the observed diagnostic features of ASD than the previously used ADOS algorithm.

The Autism Diagnostic Interview, Revised (ADI-R) ${ }^{24}$ was administered to the parents of two of the subjects with atypical deletions who were unavailable for testing with the ADOS.

The data from the Social Communication Questionnaire, cognitive testing, VABS-II, and the ADOS or ADI-R for each subject were reviewed (by N.A.). Using a clinical best estimate diagnostic approach following criteria in the Diagnostic and Statistical Manual of Mental Disorders, Fifth Edition, ${ }^{25}$ and following a method used in previous published research studies that includes clinical judgment, ${ }^{23,26,27}$ each case was classified as ASD or non-ASD.

Genetic testing. Blood samples from each patient underwent aCGH as described previously by Signature Genomics (www. signaturegenomics.com) at the time of the conference, before the study by a Clinical Laboratory Improvement Admendmentscertified laboratory ordered by the patient's local primary care physician or geneticist, or in the laboratory of Dr. Orsetta Zuffardi, Department of Medical Genetics, University of Pavia, Italy, as described previously. ${ }^{28}$

Samples tested by the Signature Genomics laboratory utilized the custom-designed OS V2.0 platform, a system that uses 135,000 oligonucleotides with approximately 10 -kb resolution in distal $11 \mathrm{q}$, thereby providing a readout of individual genes that are deleted in distal 11q.

\section{RESULTS}

Individual behavioral and genetic test results and are shown in Table 1. Of the 17 subjects in the main cohort, 8 (47\%) were determined to meet criteria for a research diagnosis of ASD. Among the male subjects, four of five (80\%) subjects who underwent complete testing met research criteria for ASD. The remaining male subject was unable to complete the ADOS, but based on overall clinical judgment he was not determined to meet criteria for ASD. Among the female subjects, 4 of 12 (33\%) met research criteria for ASD. All three of the patients with atypical deletions ( 2 male, 1 female) met criteria for ASD.

As shown in Table 1, the deletion sizes of the eight patients with ASD ranged from 8.7 to $14.6 \mathrm{Mb}$. These results indicate incomplete penetrance and no correlation between the deletion size and autism. Of note, additional copy number variants (CNVs) outside of $11 \mathrm{q}$, up to $\sim 500 \mathrm{~kb}$, were identified, and all were considered not to be of clinical significance based on their presence in the Signature Genomics database consisting of more than 60,000 normal controls and/or in the Database 


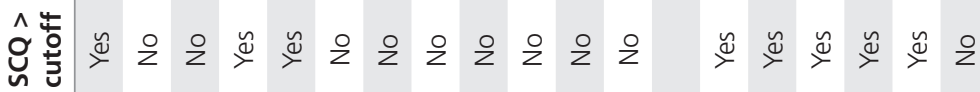

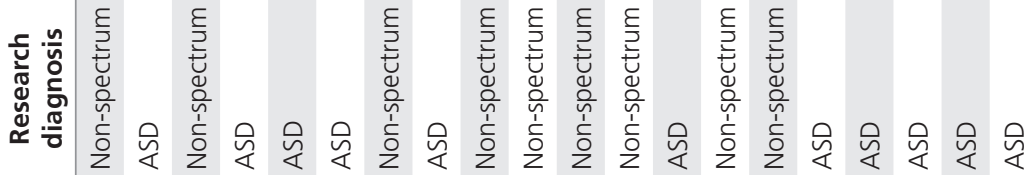

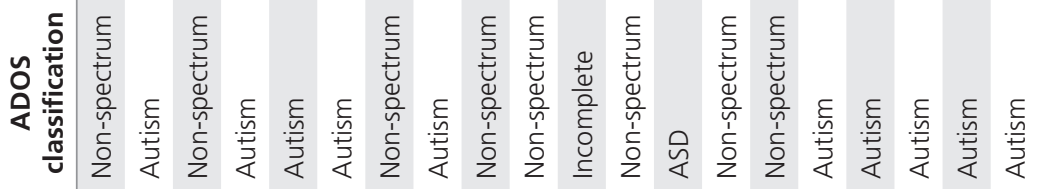

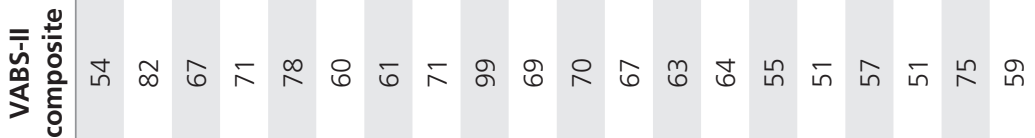

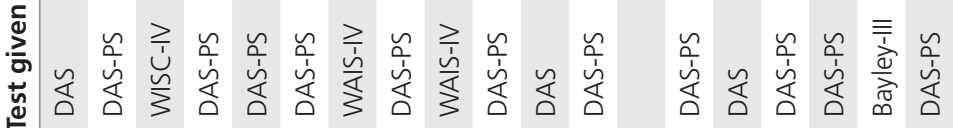

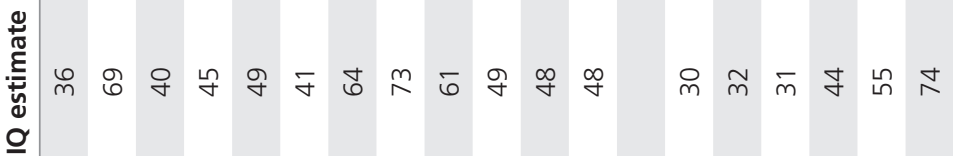

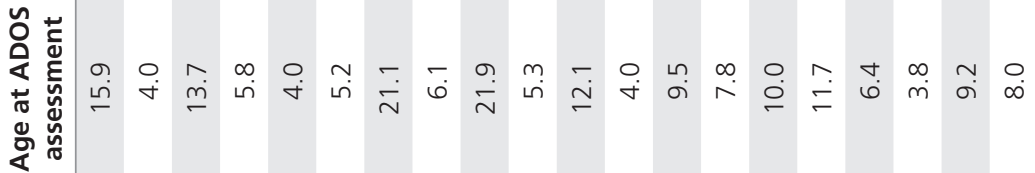

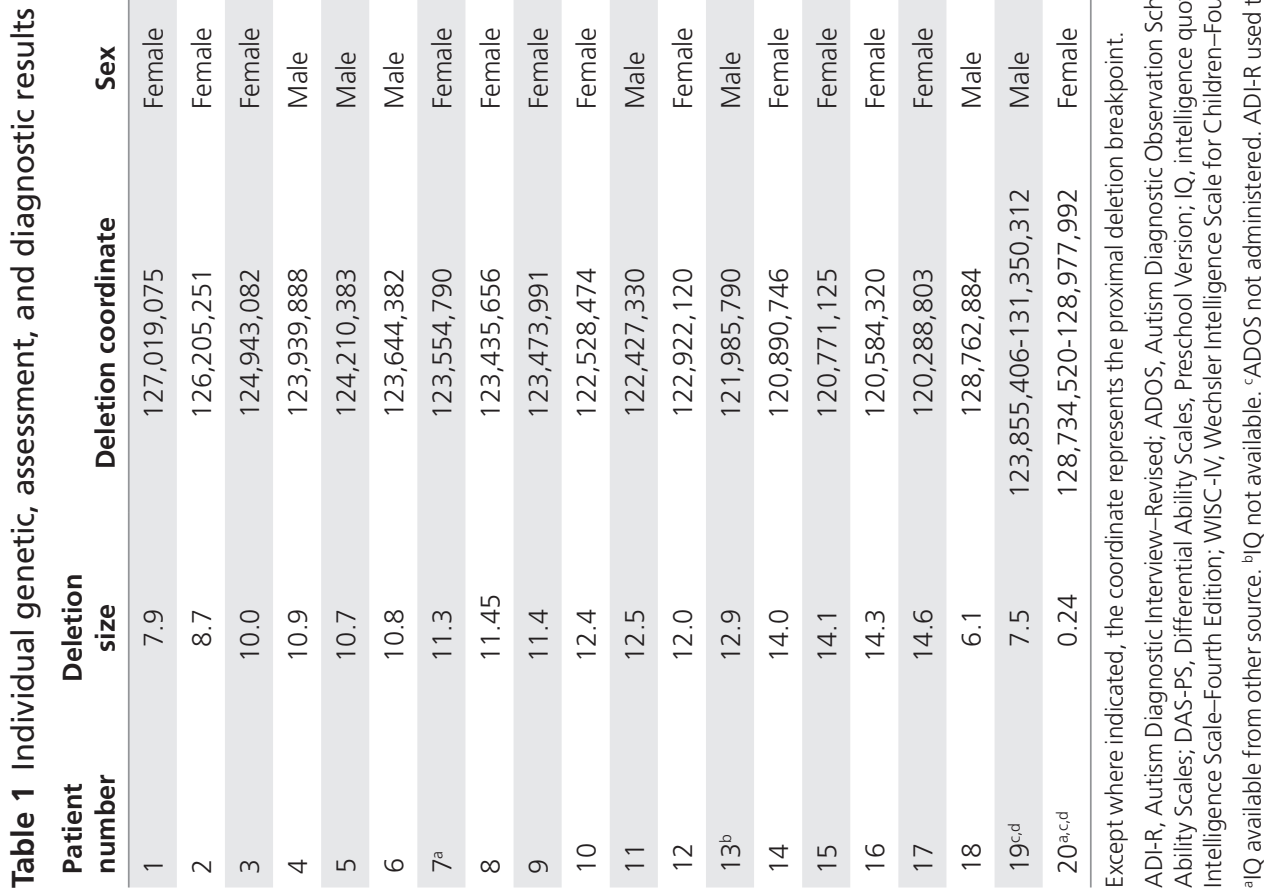




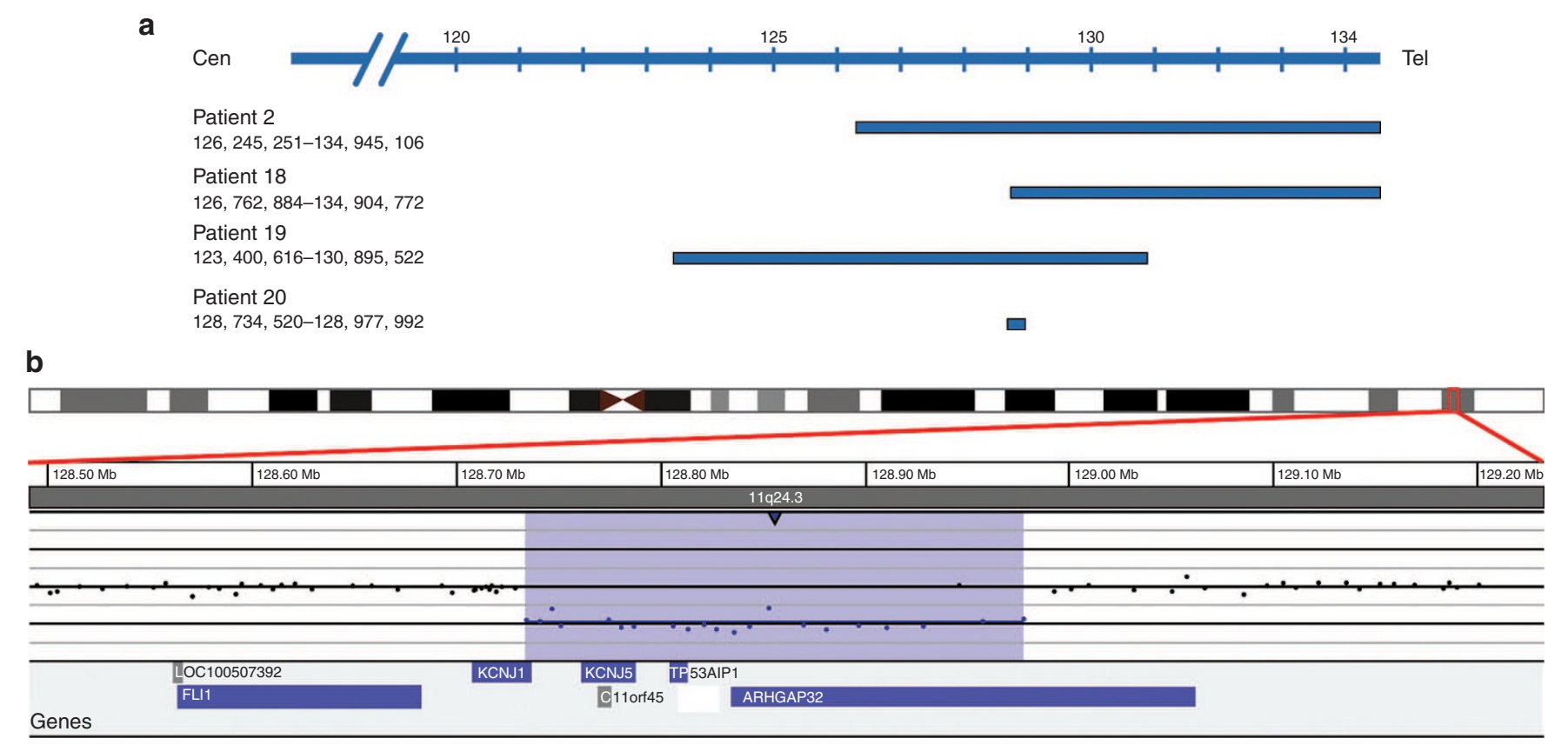

Figure 1 Deletion breakpoints of select patients. (a) Schematic demonstrating smallest size terminal 11q deletion in association with autism spectrum disorder (ASD) from the 17-patient cohort, and overlapping atypical deletions of patients with ASD in distal 11q. (b) Enlargement of 243-kb deleted region in patient 20, containing five genes, deleted in all other patients described in the current study.

of Genomic Variants (http://projects.tcag.ca/variation/). Furthermore, there was no enrichment for these CNVs in the patients with ASD. Taken together, these data implicate at least one predisposing gene for autism in the distal $8.7 \mathrm{Mb}$ of $11 \mathrm{q}$.

The three additional patients with "atypical" $11 \mathrm{q}$ deletions (Figure 1) all had autistic features. The first, patient 18, originally had autism diagnosed in his local community and subsequently underwent aCGH, revealing a $6.1-\mathrm{Mb}$ terminal deletion in 11q. We performed a cognitive and behavioral evaluation of this patient, as described above, confirming the diagnosis of ASD. His mother has mild intellectual disability; there was no evidence to suggest that she met diagnostic criteria for ASD, but this was not formally evaluated. Patient 19 had ASD diagnosed previously at a university medical center, which we confirmed with parent questionnaires and the ADI-R parent interview. He was found to have a 7.3-Mb interstitial deletion in distal 11q. This deletion overlaps with that of patient 18 across a $2.7-\mathrm{Mb}$ region. Patient 20 was identified through the UNIQUE rare chromosomal database (www.rarechromo.org). This patient had a diagnosis of intellectual disability, and a previous aCGH analysis revealed a tiny $(310 \mathrm{~kb})$ de novo microdeletion contained within the region of overlap with patients 18 and 19. Based on the results from parent questionnaires, review of previous records, and the ADI-R parent interview, we determined that this patient met criteria for ASD. We subsequently performed a higher-resolution aCGH analysis (Signature Genomics) that revealed the presence of a de novo $243-\mathrm{kb}$ interstitial deletion containing four annotated genes in the OMIM database: KCJN1, KCJN5, TP53AIP1, and ARHGAP32 (RICS).

\section{DISCUSSION}

This is the first prospective study designed to identify ASD in patients with JBS. Our initial observations verified this to be true for some patients, and we found that a large proportion of the patients met research criteria for ASD using standardized parent report and observational diagnostic measures. Although elevated rates of ASD have also been reported in other chromosomal deletion syndromes, a recent study of children with 22q11.2 deletion syndrome ${ }^{29}$ illustrates the importance of using a parent report measure as well as a standardized observation tool to avoid false identification of ASD. The ADOS and the Social Communication Questionnaire have been shown to have good sensitivity and specificity, but the reliability of diagnosis is significantly improved when both are used in combination. The patients with JBS who met criteria for ASD in the current study presented with deficits in social communication and interactions as well as repetitive and restricted behavior and interests that were not accounted for by intellectual disability.

Our results support two previous case reports in the literature suggesting that autism occurs in a subset of patients with JBS. Fisch et al. ${ }^{9}$ assessed nine patients with JS using the Childhood Autism Rating Scale and determined three scored above the cutoff for autism. Guerin et al. ${ }^{10}$ described a single patient with a $2.89-\mathrm{Mb}$ deletion in distal $11 \mathrm{q}$ who had autistic features and proposed the Kirrel3 gene as a candidate for causing autism in the patient. ASD results from a large variety of genetic causes ${ }^{30,31}$ and the results from the current study add to the growing list of genetic syndromes associated with a proportion of children who have ASD. ${ }^{32}$ 
Autism is associated with a strong sex bias (estimated 4:1 males:females), and our results are consistent with this. The males in our sample were highly likely $(80 \%)$ to meet criteria for ASD, with a frequency much higher than that in the general population. In addition, given that four of the eight individuals with ASD in the main cohort were females, and that the rate of ASD was $33 \%$ in females, the results of this study suggest that females with JBS are also at higher risk for ASD than expected in the general population. Although preliminary, these results suggest that in addition to intellectual disability, children with JBS may present with additional behavioral difficulties that further affect daily function and may warrant early intervention. All young children with JBS should be carefully screened for ASD and followed up in order to provide appropriate intervention services.

Our results demonstrated that there was no correlation between deletion size and a diagnosis of ASD, implicating at least one gene in the distal $8.7 \mathrm{Mb}$ of $11 \mathrm{q}$ that predisposes individuals to exhibit ASD with incomplete penetrance. This region contains more than 50 annotated genes, many of which are expressed in the brain. In addition to the prospective cohort of 17 patients identified, we also assessed three patients with atypical deletions in distal 11q (Figure 1). The first had an original diagnosis of autism and subsequently underwent an array CGH analysis, revealing the presence of a $6.1-\mathrm{Mb}$ terminal deletion in 11q. The second patient also had autism previously diagnosed and had a 7.3-Mb interstitial deletion in distal 11q. These two patients shared a $2.7-\mathrm{Mb}$ region of overlap, implicating the possibility of a single predisposing gene within this region for both patients. Remarkably, we identified a third patient with intellectual disability who reportedly had a tiny interstitial deletion within this $2.7-\mathrm{Mb}$ region of overlap. Results from the ADI-R parent interview from this patient were consistent with autism. This $243-\mathrm{kb}$ region of overlap, deleted in all 17 patients in our cohort, contains four annotated genes: KCNJ1, KCNJ5, TP53AIP1, and ARHGAP32.

Homozygous mutations and deletions in the KCNJ1 gene are associated with autosomal recessive Bartter syndrome. ${ }^{33}$ Individuals with heterozygous deletions of KCNJ1 are generally asymptomatic carriers for Bartter syndrome. Mutations, but not deletions, in the KCNJ5 gene have been reported in autosomal dominant hyperaldosternoism type III and, in one family, autosomal dominant long QT syndrome. ${ }^{34}$ TP53AIP1 is thought to play an important role in mediating $\mathrm{p} 53$-dependent apoptosis. ${ }^{35}$ ARHGAP32 (formerly RICS) encodes a neuronassociated GTPase-activating protein that regulates dendritic spine morphology and strength by modulating Rho GTPase. ${ }^{31,36}$ Studies of mice revealed that ARHGAP32 is involved in early brain development, including extension of axons and dendrites, and postnatal remodeling and fine-tuning of neural circuits. ${ }^{37}$ To date, nothing is known about the role of ARHGAP32 in cognition or behavior. Studies in mice have demonstrated that of these four genes, only KCNJ5 and ARHGAP32 are expressed in the brain. ${ }^{36,38}$
Interestingly, the 243 -kb region deleted in patient 20 also overlaps the distal end of the $2.89-\mathrm{Mb}$ interstitial region reported by Guerin et al. ${ }^{10}$ in a patient with features of JBS and autism. Although these authors proposed the Kirrel3 gene as a candidate gene for autism in their patient, our findings of ASD in patients without deletion of Kirrel3 suggest that this gene is not causal in at least a subset of patients with distal 11q deletions. Consequently, our data implicate at least one other predisposing gene in distal 11q for autism. We propose ARHGAP32 as a candidate gene for causing ASD in at least a subset of patients with distal $11 \mathrm{q}$ deletions. Further support for ARHGAP32 as a candidate gene is provided by previous studies implicating susceptibility loci containing genes that encode proteins that are involved in GTPase signaling in the brain. ${ }^{39}$

The incomplete penetrance of ASD suggests the presence of additional genetic, epigenetic, and/or environmental modifiers that can suppress the development of autism in patients with JBS. Interestingly, Girirajan et $\mathrm{al}^{40}$ recently proposed a two-hit model for severe psychiatric illness in patients carrying 16p.12.1 deletions. Specifically, they identified an additional CNV of possible clinical significance in $30 \%$ of these patients. In most cases, the 16p.12.1 deletion was inherited from a clinically unaffected parent, consistent with a two-hit model. In the same study, these authors analyzed other patients with syndromes that largely result from de novo deletions, including Williams, Smith-Magenis, and DiGeorge syndromes. In those cases, very few or no second-hit disease-causing CNVs were identified. Our findings suggest that in JBS, it is likely that there are additional genetic variants other than CNVs (e.g., single-nucleotide polymorphisms) that contribute to the ASD phenotype, along with other epigenetic and environmental factors. However, it is still possible that microscopic CNVs involving additional loci not detected in our patient cohort may be necessary in combination with an 11q deletion to manifest the ASD phenotype. .0,41 $^{40}$ Future studies will be aimed at studying genetically engineered ARHGAP32 knockout mice for cognitive impairment and autistic features, as well as studying neurons derived from induced pleuripotent stem cells from 11q- patients. In addition, further studies are needed to examine sex-related frequency differences.

There are several limitations to this study that should be considered. Given the limited sample size, it is premature to estimate the prevalence of ASD in patients with JBS. It can be challenging to accurately make a diagnosis of ASD in the presence of intellectual disability. In this sample, however, there did not appear to be a clear relationship between level of intellectual disability and ASD. This group of subjects may represent a biased sample of patients with JBS. Most of the subjects were from families who had contact with the 11q Research and Resource Group and traveled to attend a biennial conference. It is possible that these patients may be more affected than average, which could lead to an overestimation of the frequency of ASD. These families were likely to have the resources available to travel to the conference, which may have also biased the sample. 


\section{ACKNOWLEDGMENTS}

We acknowledge the families who graciously participated in our research and the assistance of the members of the Center for Behavioral Teratology at San Diego State University, particularly Delilah Bolo, Genevive Brusati, Nicole Crocker, Benjamin Deweese, Jason Dudley, Leila Glass, Diana Graham, Heather Holden, Kristina Hubbard, Christie McGee, Kimberly Ogle, Danielle Sandner, Jill Vander Velde, Linnea Vaurio, and Amanda Wagner, who provided assistance with data collection. The support of Center Director Edward Riley and Jill Mokry from Signature Genomics was very helpful in the interpretation of some of the aCGH analyses. We also acknowledge Teresa Mattina and Orsetta Zuffardi for performing aCGH analysis of several of the patients. An intramural grant from the University of California, San Diego Department of Pediatrics, and the generous support of the cast and crew of the television show "How I Met Your Mother" provided funding for this work.

\section{DISCLOSURE}

The authors declare no conflict of interest.

\section{REFERENCES}

1. Jacobsen $P$, Hauge M, Henningsen $K$, Hobolth N, Mikkelsen M, Philip J. An $(11 ; 21)$ translocation in four generations with chromosome 11 abnormalities in the offspring. A clinical, cytogenetical, and gene marker study. Hum Hered 1973;23:568-585.

2. Mattina T, Perrotta CS, Grossfeld P. Jacobsen syndrome. Orphanet J Rare Dis 2009;4:9.

3. Grossfeld PD, Mattina T, Lai Z, et al. The 11q terminal deletion disorder: a prospective study of 110 cases. Am J Med Genet A 2004;129A:51-61.

4. Lee WB, O'Halloran HS, Grossfeld PD, Scher C, Jockin YM, Jones C. Ocular findings in Jacobsen syndrome. J AAPOS 2004;8:141-145.

5. Haghi M, Dewan A, Jones KL, Reitz R, Jones C, Grossfeld P. Endocrine abnormalities in patients with Jacobsen (11q-) syndrome. Am J Med Genet $A$ 2004;129A:62-63.

6. Maas AP, Grossfeld PD, Didden R, et al. Sleep problems in individuals with $11 \mathrm{q}$ terminal deletion disorder (Jacobsen syndrome). Genet Couns 2008;19:225235.

7. Coldren CD, Lai Z, Shragg P, et al. Chromosomal microarray mapping suggests a role for BSX and Neurogranin in neurocognitive and behavioral defects in the $11 \mathrm{q}$ terminal deletion disorder (Jacobsen syndrome). Neurogenetics 2009;10:89-95.

8. Bernaciak J, Szczałuba K, Derwińska K, et al. Clinical and molecularcytogenetic evaluation of a family with partial Jacobsen syndrome without thrombocytopenia caused by an approximately $5 \mathrm{Mb}$ deletion del(11)(q24.3). Am J Med Genet A 2008;146A:2449-2454.

9. Fisch GS, Grossfeld P, Falk R, Battaglia A, Youngblom J, Simensen R. Cognitivebehavioral features of Wolf-Hirschhorn syndrome and other subtelomeric microdeletions. Am J Med Genet C Semin Med Genet 2010;154C:417-426.

10. Guerin A, Stavropoulos DJ, Diab Y, et al. Interstitial deletion of 11q-implicating the KIRREL3 gene in the neurocognitive delay associated with Jacobsen syndrome. Am J Med Genet A 2012;158A:2551-2556.

11. Ye M, Hamzeh R, Geddis A, Varki N, Perryman MB, Grossfeld P. Deletion of JAM$C$, a candidate gene for heart defects in Jacobsen syndrome, results in a normal cardiac phenotype in mice. Am J Med Genet A 2009;149A:1438-1443.

12. Favier $R$, Jondeau $K$, Boutard $P$, et al. Paris-Trousseau syndrome: clinical, hematological, molecular data of ten new cases. Thromb Haemost 2003:90:893-897.

13. Rutter M, Bailey A, Lord C. Social Communication Questionnaire. Western Psychological Services: Los Angeles, CA, 2003.

14. Corsello C, Hus V, Pickles A, et al. Between a ROC and a hard place: decision making and making decisions about using the SCQ. J Child Psychol Psychiatry 2007:48:932-940.
15. Sparrow SS, Cicchetti DV, Balla DA. Vineland Adaptive Behavior Scales. II. Survey Forms Manual, 2nd edn. American Guidance Service: Circle Pines, MN, 2005.

16. Lord C, Risi S, DiLavore PS, Shulman C, Thurm A, Pickles A. Autism from 2 to 9 years of age. Arch Gen Psychiatry 2006;63:694-701.

17. Bayley N. Bayley Scales of Infant and Toddler Development, Third Edition (Bayley-III), 3rd edn. Harcourt Brace \& Co: San Antonio, TX, 2005.

18. Elliott CD. Differential Ability Scales. Psychological Corporation: San Antonio, TX, 1990.

19. Wechsler D. Wechsler Intelligence Scale for Children-Fourth Edition. The Psychological Corporation: San Antonio, TX, 2003.

20. Wechsler D. Wechsler Adult Intelligence Scale_Fourth Edition. Pearson Assessment: San Antonio, TX, 2008.

21. Lord C, Rutter M, DiLavore PC, Risi S. Autism Diagnostic Observation Schedule. Western Psychological Services: Los Angeles, CA, 2001.

22. Gotham K, Risi S, Pickles A, Lord C. The Autism Diagnostic Observation Schedule: revised algorithms for improved diagnostic validity. J Autism Dev Disord 2007;37:613-627.

23. Gotham K, Risi S, Dawson G, et al. A replication of the Autism Diagnostic Observation Schedule (ADOS) revised algorithms. J Am Acad Child Adolesc Psychiatry 2008;47:642-651.

24. Rutter M, Le Couteur A, Lord C. ADI-R. Autism Diagnostic Interview-Revised. WPS Edition. Western Psychological Services: Los Angeles, CA, 2003.

25. American Psychiatric Association. Diagnostic and Statistical Manual of Mental Disorders, 5th edn. American Psychiatric Publishing: Arlington, VA, 2013.

26. Mahoney WJ, Szatmari P, MacLean JE, et al. Reliability and accuracy of differentiating pervasive developmental disorder subtypes. J Am Acad Child Adolesc Psychiatry 1998;37:278-285.

27. Risi S, Lord C, Gotham K, et al. Combining information from multiple sources in the diagnosis of autism spectrum disorders. J Am Acad Child Adolesc Psychiatry 2006;45:1094-1103.

28. Iascone M, Ciccone R, Galletti L, et al. Identification of de novo mutations and rare variants in hypoplastic left heart syndrome. Clin Genet 2012;81:542-554.

29. Angkustsiri K, Goodlin-Jones B, Deprey L, Brahmbhatt K, Harris S, Simon TJ. Social impairments in chromosome 22q11.2 deletion syndrome (22q11.2DS): autism spectrum disorder or a different endophenotype? J Autism Dev Disord 2014;44:739-746.

30. Geschwind DH. Autism: many genes, common pathways? Cell 2008;135:391395.

31. Zeidán-Chuliá F, Rybarczyk-Filho JL, Salmina AB, de Oliveira BH, Noda M, Moreira JC. Exploring the multifactorial nature of autism through computational systems biology: calcium and the Rho GTPase RAC1 under the spotlight. Neuromolecular Med 2013;15:364-383.

32. Levy D, Ronemus M, Yamrom B, et al. Rare de novo and transmitted copynumber variation in autistic spectrum disorders. Neuron 2011;70:886-897.

33. International Collaborative Study Group for Bartter-like Syndromes. Mutations in the gene encoding the inwardly-rectifying renal potassium channel, ROMK, cause the antenatal variant of Bartter syndrome: evidence for genetic heterogeneity. Hum Mol Genet 1997;6:17-26.

34. Choi M, Scholl UI, Yue P, et al. K+ channel mutations in adrenal aldosteroneproducing adenomas and hereditary hypertension. Science 2011;331: 768-772.

35. Oda K, Arakawa H, Tanaka T, et al. p53AIP1, a potential mediator of p53dependent apoptosis, and its regulation by Ser-46-phosphorylated p53. Cell 2000;102:849-862.

36. Nakamura T, Komiya M, Sone K, et al. Grit, a GTPase-activating protein for the Rho family, regulates neurite extension through association with the TrkA receptor and $\mathrm{N}-\mathrm{Shc}$ and $\mathrm{CrkL/Crk}$ adapter molecules. Mol Cell Biol 2002;22:8721-8734.

37. Nasu-Nishimura Y, Hayashi T, Ohishi T, et al. Role of the Rho GTPase-activating protein RICS in neurite outgrowth. Genes Cells 2006;11:607-614.

38. Perry CA, Pravetoni M, Teske JA, et al. Predisposition to late-onset obesity in GIRK4 knockout mice. Proc Natl Acad Sci USA 2008;105:8148-8153.

39. Geschwind DH. Genetics of autism spectrum disorders. Trends Cogn Sci 2011;15:409-416.

40. Girirajan S, Rosenfeld JA, Cooper GM, et al. A recurrent 16p12.1 microdeletion supports a two-hit model for severe developmental delay. Nat Genet 2010;42:203-209.

41. Girirajan S, Rosenfeld JA, Coe BP, et al. Phenotypic heterogeneity of genomic disorders and rare copy-number variants. N Engl J Med 2012;367: $1321-1331$. 